\title{
Delirium como fator de risco para demência em idosos: uma atualização
}

Delirium as risk factor for dementia in older adults: an update

Natália Mota S. Chagas', Daniel G. Suzuki Borges', Marcos Hortes N. Chagas²

RESUMO

Objetivo: Realizar uma revisão atualizada dos estudos que avaliaram o delirium como fator de risco para o desenvolvimento de demência em idosos. Métodos: A revisão foi realizada utilizando-se a base de dados do PubMed, com o seguinte cruzamento de descritores: risk factors and (delirium or acute confusional state) and dementia. Apenas estudos do tipo coorte publicados entre janeiro de 2000 a maio de 2015 foram considerados. Resultados: Foram selecionados oito estudos de acordo com os critérios de inclusão e exclusão. Todos os estudos apontaram para um aumento do risco de demência ou declínio cognitivo após a ocorrência de delirium, apesar das diferenças entre os estudos em relação à causa do deli-

\section{Palavras-chave}

Demência, delirium, fatores de risco.

\section{Keywords}

Dementia, delirium, risk factors.

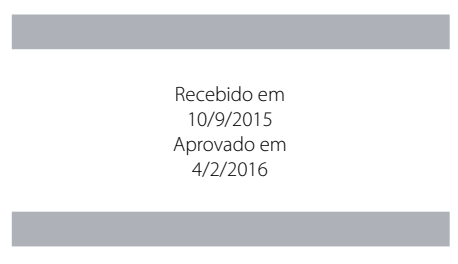

DOI: $10.1590 / 0047-2085000000109$ rium. Conclusões: Os estudos atuais apontam para uma clara associação entre delirium e aumento do risco de demência. Dessa forma, psiquiatras e médicos generalistas precisam ficar atentos aos indícios de declínio cognitivo após a ocorrência do delirium em idosos.

\section{ABSTRACT}

Objective: To perform an update review of the studies that evaluated delirium as a risk factor for developing dementia in older adults. Methods: A review was performed using PubMed database, crossing the following descriptors: risk factors and (delirium or acute confusional state) and dementia. Only cohort studies published from January 2000 to May 2015 were considered. Results: We selected eight studies according to the inclusion and exclusion criteria. All studies pointed to an increased risk of dementia or cognitive decline after the occurrence of delirium, despite the differences between the studies in relation to the cause of delirium. Conclusions: Recent studies indicate a clear association between delirium and increased risk of dementia. Therefore, the psychiatrists and general practitioners need to pay attention to the evidence of cognitive decline after the occurrence of delirium in older adults.

\footnotetext{
1 Universidade de São Paulo (USP), Faculdade de Medicina de Ribeirão Preto (FMRP), Departamento de Neurociências e Ciências do Comportamento

2 Universidade Federal de São Carlos (UFSCar), Centro de Ciências Biológicas e da Saúde, Departamento de Gerontologia.
}

Endereço para correspondência: Marcos Hortes N. Chagas

Universidade Federal de São Carlos,

Centro de Ciências Biológicas e da Saúde,

Departamento de Gerontologia

Rodovia Washington Luís, km 235

13565-905 - São Carlos, SP

Telefone: (55 16) 3306-6938

E-mail: setroh@hotmail.com; mchagas@ufscar.br 


\section{INTRODUÇÃO}

O delirium ou estado confusional agudo é caracterizado por um quadro de início súbito no qual ocorre alteração das funções mentais, com alteração no nível de consciência, desatenção e prejuízo na memória', além da possibilidade de comprometimento comportamental e do pensamento ${ }^{2}$.

É uma síndrome altamente prevalente em idosos admitidos em ambiente hospitalar, com variação entre 7\% e 52\% ${ }^{3}$. Essa ampla variação pode ser explicada pela heterogeneidade dos grupos selecionados nos estudos em relação à faixa etária, métodos diagnósticos, comorbidades associadas, entre outros, como diferenças entre sexos e fatores precipitantes associados.

O delirium geralmente é sub-reconhecido, o que pode implicar complicações graves e desfechos negativos, como longo período de hospitalização, aumento do risco de complicações e altas taxas de mortalidade ${ }^{46}$.

Considerando os mecanismos neurobiológicos envolvidos, acredita-se que múltiplos fatores levem a uma via final com excesso de dopamina e depleção colinérgica. A inflamação sistêmica parece afetar o sistema nervoso central por meio de diversas vias, o que comprometeria seu funcionamento adequado e, de alguma forma, acarretaria uma depleção colinérgica, contribuindo para disfunção cognitiva 4 . Além disso, a relação entre processos inflamatórios e neurodegenerativos tem um papel importante na associação entre delirium e demência ${ }^{7}$, a qual parece ser bidirecional ${ }^{8}$.

Sabe-se que inúmeros estudos demonstraram maior risco de delirium em pacientes com demência9. Adicionalmente, nos últimos anos, diversos estudos têm apontado também para o sentido inverso, de forma que o estado confusional agudo também poderia ser um fator de risco para o desenvolvimento de demência9,10. Considerando essa relação próxima entre delirium e demência, parece pertinente realizar uma atualização relacionada a esse importante tema, incluindo os estudos realizados nos últimos 15 anos.

\section{OBJETIVO}

O objetivo da revisão é compilar uma atualização dos estudos da associação entre a ocorrência de delirium como fator de risco para o desenvolvimento de demência.

\section{MÉTODOS}

A revisão foi realizada utilizando-se a base de dados do PubMed, com o seguinte cruzamento de palavras-chave: riskfactors and (delirium or acute confusional state) and dementia.

Os critérios de inclusão foram artigos em inglês, com delineamento do tipo coorte, realizados em humanos e publicados entre janeiro de 2000 e maio de 2015. Estudos realizados em indivíduos com idade inferior a 60 anos e que avaliaram delirium com quadro demencial sobreposto foram excluídos da análise. Um total de 244 artigos foi encontrado; desses, foram selecionados oito artigos de acordo com os critérios de inclusão e exclusão.

\section{RESULTADOS}

Todos os artigos que foram selecionados nesta revisão apontaram para um aumento significativo no risco de desenvolver demência ou declínio cognitivo em pacientes com história de delirium comparados com aqueles sem delirium, conforme observado na Tabela 1. A amostra total de participantes dos estudos variou de 50 a 533 idosos ${ }^{11,12}$.

Em quatro dos artigos selecionados, a amostra avaliada foi referente a pacientes internados por causa da necessidade de procedimentos cirúrgicos ortopédicos ${ }^{3,10,13,14}$. Entre esses quatro estudos, a média de observação e reavaliação para o diagnóstico de demência variou entre seis meses ${ }^{3} \mathrm{e}$ cinco anos ${ }^{13}$. Dois estudos fizeram o seguimento apenas de idosos sem demência ${ }^{3,13}$, e os dados de um dos estudos ${ }^{14}$ foram coletados como parte de um projeto que envolvia um ensaio clínico randomizado de dose baixa de haloperidol.

Em outros quatro estudos 2,11,12,15, a relação entre delirium e demência foi avaliada na população idosa sem associação a condições ortopédicas. Em dois deles ${ }^{12,15}$, as pesquisas foram baseadas na mesma amostra de 553 idosos de 85 anos ou mais moradores de uma cidade finlandesa, o que representava $92 \%$ da população dessa faixa etária. O estudo de Rahkonen et al..$^{15}$ seguiu apenas os idosos sem demência na linha de base $(n=366)$. O estudo de Davis et al..$^{12}$ avaliou essa amostra em quatro momentos diferentes (3, 5, 8 e 10 anos) e é o único entre os estudos selecionados que utilizou biomarcadores na avaliação diagnóstica. O estudo de van Rijsbergen et al. ${ }^{11}$ avaliou apenas idosos após a ocorrência de acidente vascular encefálico e McCusker et al. ${ }^{2}$ avaliaram idosos admitidos na emergência de um hospital universitário.

Em relação aos critérios utilizados para o diagnóstico de delirium e demência, seis estudos ${ }^{2,3,12-15}$ relataram o uso dos critérios do DSM-III-R ou DSM-IV (Manual Diagnóstico e Estatístico dos Transtornos Mentais, 3a edição revisada e $4^{a}$ edição). O instrumento Confusion Assessment Method ${ }^{2,3,10,11,14}$ e o Miniexame do Estado Mental (MMSE) 2,10,12-15 foram os mais utilizados para avaliação de delirium e demência/declínio cognitivo, respectivamente.

\section{DISCUSSÃO}

Todos os estudos selecionados nesta revisão apontaram para o delirium como fator de risco para o desenvolvimento de demência ou declínio cognitivo, independentemente do 
tempo de seguimento realizado por cada estudo. Esse achado reforça a importância de investigar a presença de declínio cognitivo e/ou demência após a ocorrência de delirium.

A maioria dos estudos utilizou o MMSE, que é um teste de rastreio cognitivo, o que pode ser uma limitação, visto que não revela os domínios cognitivos com mais prejuízo.
Apenas o estudo de van Rijsbergen et al. ${ }^{11}$ avaliou os domínios cognitivos de forma mais específica por meio do CAMCOG (Cambridge Cognitive Examination), uma bateria multidimensional, e encontrou que os domínios cognitivos mais afetados foram memória, linguagem, habilidade visuoespacial e funcionamento executivo.

Tabela 1. Estudos de coorte que avaliaram delirium como fator de risco para desenvolvimento de demência

\begin{tabular}{|c|c|c|c|c|c|}
\hline Estudo & N & Tempo de seguimento & Avaliação & Resultados & Conclusões \\
\hline Davis et al., 2012 & $\begin{array}{l}553 \text { idosos } \\
\geq 85 \text { anos }\end{array}$ & $3,5,8$ e 10 anos & $\begin{array}{l}\text { 1. Biomarcadores } \\
\text { neuropatológicos a partir de } \\
\text { autópsia cerebral } \\
\text { 2. Avaliação médica/ } \\
\text { neurológica } \\
\text { 3. Demência: DSM-III-R, } \\
\text { MMSE, IADL, entrevistas e } \\
\text { revisão de prontuários }\end{array}$ & $\begin{array}{l}\text { 1. Aumento do risco da } \\
\text { incidência de demência (OR= } \\
8,7 ; \text { IC } 95 \% 2,1-35,0) \\
\text { 2. Delirium associado com } \\
\text { piora da gravidade da } \\
\text { demência (OR=3,1; IC } 95 \% \\
1,5-6,3 \text { ) e maior prejuízo } \\
\text { no funcionamento global } \\
\text { (OR=2,8; IC } 95 \% 1,5-5,5)\end{array}$ & $\begin{array}{l}\text { Delirium é um importante } \\
\text { fator de risco para incidência } \\
\text { de demência e declínio } \\
\text { cognitivo em idosos mais } \\
\text { velhos }\end{array}$ \\
\hline $\begin{array}{l}\text { Krogseth et al., } \\
2011^{3}\end{array}$ & 106 idosos & $\begin{array}{l}6 \text { meses após fratura de } \\
\text { quadril }\end{array}$ & $\begin{array}{l}\text { 1. Delirium: Confusion } \\
\text { Assessment Method } \\
\text { 2. Declínio cognitivo pré- } \\
\text {-fratura: IQCODE-Short Form } \\
\text { 3. Demência: DSM-IV e testes } \\
\text { neuropsicológicos }\end{array}$ & $\begin{array}{l}\text { 1. Delirium foi um forte } \\
\text { preditor de demência (OR= } \\
\text { 10,5; IC } 95 \% \text { 1,6-70,3) } \\
\text { 2. IQCODE-SF não apresentou } \\
\text { associação com a incidência de } \\
\text { demência }\end{array}$ & $\begin{array}{l}\text { Delirium é o preditor mais } \\
\text { importante relacionado com a } \\
\text { incidência de demência após } \\
6 \text { meses de uma fratura de } \\
\text { quadril }\end{array}$ \\
\hline $\begin{array}{l}\text { van Rijsbergen et } \\
\text { al., 201111 }\end{array}$ & $\begin{array}{l}50 \text { idosos com AVE (22 com e } \\
28 \text { sem delirium) }\end{array}$ & 2 anos do AVE & $\begin{array}{l}\text { 1. Demência: CDR e CAMCOG } \\
\text { 2. Delirium: Confusion } \\
\text { Assessment Method } \\
\text { 3. Declínio cognitivo prévio: } \\
\text { IQCODE }\end{array}$ & $\begin{array}{l}\text { 1. Delirium foi um preditor } \\
\text { independente de demência } \\
\text { (OR }=4,7 ; \text {; } 95 \% 1,1-20,4) \\
\text { 2. Os domínios cognitivos } \\
\text { mais prejudicados foram } \\
\text { memória, linguagem, } \\
\text { habilidade visuoespacial e } \\
\text { funcionamento executivo }\end{array}$ & $\begin{array}{l}\text { Delirium na fase aguda } \\
\text { pós-AVE é fator de risco } \\
\text { importante de demência }\end{array}$ \\
\hline Bickel et al., $2008^{10}$ & $\begin{array}{l}200 \text { idosos submetidos à } \\
\text { cirurgia de quadril }\end{array}$ & $\begin{array}{l}8 \text { a } 38 \text { meses após alta } \\
\text { hospitalar }\end{array}$ & $\begin{array}{l}\text { 1. Delirium: Confusion } \\
\text { Assessment Method } \\
\text { 2. Demência: MMSE, CDR }\end{array}$ & $\begin{array}{l}\text { 1. Alta associação entre } \\
\text { delirium e prejuízo cognitivo } \\
(0 \mathrm{R}=41,2 ; \text { IC } 95 \% \text { 4,3-396,2) }\end{array}$ & $\begin{array}{l}\text { Delirium é preditor de } \\
\text { declínio cognitivo com maior } \\
\text { incidência de necessidade de } \\
\text { cuidados }\end{array}$ \\
\hline Kat et al., $2008^{14}$ & $\begin{array}{l}112 \text { idosos } \\
\geq 70 \text { anos submetidos à } \\
\text { cirurgia de quadril }\end{array}$ & 30 meses após alta hospitalar & $\begin{array}{l}\text { 1. Delirium: DSM-IV, Confusion } \\
\text { Assessment Method } \\
\text { 2. Demência: DSM-IV, MMSE, } \\
\text { Digit Span test }\end{array}$ & $\begin{array}{l}\text { 1. Delirium foi associado com } \\
\text { diagnóstico de demência ou } \\
\text { comprometimento cognitivo } \\
\text { leve ( } R R=1,9 ; \text { IC } 95 \% 1,1-3,3 \text { ) } \\
\text { 2. Delirium foi associado com } \\
\text { aumento da mortalidade (RR } \\
=1,6 ; \text { IC } 95 \% 1,0-2,6)\end{array}$ & $\begin{array}{l}0 \text { risco de demência ou } \\
\text { comprometimento cognitivo } \\
\text { leve é quase o dobro em } \\
\text { idosos que apresentaram } \\
\text { delirium após cirurgia de } \\
\text { quadril comparados com } \\
\text { pacientes sem delirium }\end{array}$ \\
\hline $\begin{array}{l}\text { Rahkonen et al., } \\
2001^{15}\end{array}$ & $\begin{array}{l}366 \text { idosos sem demência } \\
\geq 85 \text { anos }\end{array}$ & 3 anos & $\begin{array}{l}\text { 1. Avaliação médica/ } \\
\text { neurológica } \\
\text { 2. Demência: DSM-III-R, } \\
\text { MMSE, IADL e entrevistas } \\
\text { 3. Delirium: DSM-III-R, } \\
\text { entrevistas clínicas e revisão } \\
\text { do prontuário }\end{array}$ & $\begin{array}{l}\text { 1. Delirium foi associado com } \\
\text { MMSE < } 24 \text { (OR = 3,4; IC } 95 \% \\
1,3-9,3) \\
\text { 2. Houve maior incidência de } \\
\text { demência entre os idosos que } \\
\text { apresentaram delirium } \\
(\mathrm{p}<0,001)\end{array}$ & $\begin{array}{l}\text { Existe associação significante } \\
\text { entre delirium e um novo } \\
\text { diagnóstico de demência }\end{array}$ \\
\hline $\begin{array}{l}\text { Lundström et al., } \\
2003^{13}\end{array}$ & $\begin{array}{l}78 \text { idosos } \\
\geq 65 \text { anos submetidos à } \\
\text { cirurgia por fratura de colo } \\
\text { do fêmur }\end{array}$ & 5 anos & $\begin{array}{l}\text { 1. Delirium: DSM-IV, Organic } \\
\text { Brain Syndrome Scale, revisão } \\
\text { de prontuário } \\
\text { 2. Demência: DSM-IV, MMSE }\end{array}$ & $\begin{array}{l}\text { 1. Delirium no pós-cirúrgico } \\
\text { foi associado com aumento no } \\
\text { desenvolvimento de demência } \\
(0 \mathrm{R}=5,7 \text { IC } 95 \% 1,3-23,6)\end{array}$ & $\begin{array}{l}\text { Delirium está associado com } \\
\text { aumento do diagnóstico de } \\
\text { demência. Outros fatores } \\
\text { associados ao aumento } \\
\text { de demência foram DM e } \\
\text { menores escores em testes } \\
\text { cognitivos na admissão }\end{array}$ \\
\hline $\begin{array}{l}\text { McCusker et al., } \\
2001^{2}\end{array}$ & $\begin{array}{l}315 \text { idosos } \\
\geq 65 \text { anos divididos em } 4 \\
\text { grupos }\end{array}$ & $\begin{array}{l}\text { 2, } 6 \text { e } 12 \text { meses após admissão } \\
\text { hospitalar }\end{array}$ & $\begin{array}{l}\text { 1. Delirium: DSM-III-R, } \\
\text { Confusion Assessment Method } \\
\text { 2. Demência: IQCODE, Barthel } \\
\text { index, IADL, MMSE }\end{array}$ & $\begin{array}{l}\text { 1. Diminuição de 3,36 (IC 95\% } \\
6,2-0,6) \text { pontos no MMSE } \\
\text { após } 12 \text { meses em idosos com } \\
\text { delirium comparados com } \\
\text { aqueles sem delirium }\end{array}$ & $\begin{array}{l}\text { Delirium é um fator preditor } \\
\text { independente para piora } \\
\text { cognitiva e funcional no } \\
\text { seguimento em idosos com ou } \\
\text { sem demência }\end{array}$ \\
\hline
\end{tabular}

AVE: acidente vascular encefálico; CAMCOG: Cambridge Cognitive Examination; CCL: comprometimento cognitivo leve; CDR: Clinical Dementia Rating Scale; DM: diabetes mellitus; DSM: Manual Diagnóstico e Estatístico dos Transtornos Mentais; IC: intervalo de confiança; IADL: Instrumental Activities of Daily Living; IQCODE: Informant Questionnaire on Cognitive Decline in the Elderly; MMSE: Miniexame do Estado Mental; 0D: odds ratio; RR: risco relativo. 
Ainda em relação aos instrumentos para diagnóstico, os critérios do DSM utilizados na maioria dos estudos são geralmente considerados padrão-ouro no diagnóstico de transtornos mentais, o que significa que o diagnóstico é essencialmente baseado em uma avaliação clínica. Dessa forma, deve-se considerar que sintomas dessas entidades podem ser similares ou mesmo sobrepostos, o que pode dificultar o diagnóstico diferencial entre elas. É interessante observar que o estudo de Davis et al. ${ }^{12}$, o único que utilizou biomarcadores na avaliação, não encontrou associação entre a história de delirium e a presença de placas neuríticas, infartos, a-sinucleinopatia e perda neuronal na substância nigra.

Apesar de não ter sido tema desta revisão, os resultados demonstraram que a ocorrência de delirium na população idosa também foi associada com pior prognóstico, com grave dependência para realização das atividades de vida diária ${ }^{10}$, piora nos sintomas de pacientes com declínio cognitivo prévio ${ }^{13}$, deterioração da função global ${ }^{2,12}$, necessidade de cuidados por mais tempo ${ }^{10}$, além de alta taxa de mortalidade ${ }^{12-14}$. Dessa forma, devem-se considerar a avaliação e o diagnóstico precoce de ambas as condições com a finalidade de evitar esses desfechos.

Entre as variáveis confundidoras, deve-se ressaltar que o perfil cognitivo antes da ocorrência de delirium é essencial na avaliação dessa associação. Dessa forma, Krogseth et al. ${ }^{3}$ buscaram avaliar a cognição dos idosos antes da ocorrência de fratura por meio do Questionário do Informante do Declínio Cognitivo em Idoso (IQCODE-SF), direcionado ao cuidador. Nesse estudo, 38\% dos pacientes que apresentaram delirium foram diagnosticados com demência após um acompanhamento por seis meses, comparados a apenas 7\% daqueles sem história de delirium, e o perfil cognitivo prévio não se associou ao aumento da incidência de demência. Outras variáveis de confusão devem ser levadas em consideração nesse tipo de desenho de estudo como idade, sexo e presença de comorbidades. Em um dos estudos selecionados, Rahkonen et al. ${ }^{15}$ encontraram que delirium estava associado com prejuízo visual, escores no MMSE menores que 24 e aumento da pressão sistólica, ressaltando a importância de avaliar possíveis variáveis de confusão e mediadoras.

Apenas um estudo ${ }^{14}$ pôde ser utilizado para avaliar o efeito do tratamento farmacológico do delirium na incidência de demência. Kat et al..$^{14}$ realizaram com parte de sua amostra um ensaio clínico randomizado comparando o uso de haloperidol em baixa dose por três dias e placebo, fruto de outra publicação ${ }^{16}$, e não encontraram diferenças nos desfechos declínio cognitivo e mortalidade no seguimento de 30 meses. Deve-se considerar nesse caso como limitação o tempo muito curto do uso de haloperidol como medida preventiva.

Todos os artigos selecionados apresentaram um desfecho compatível com a associação direta entre delirium e prejuízo cognitivo. Entretanto, uma pergunta que permanece sem resposta é se o delirium poderia ser uma causa de demência ou se apenas contribui para o desenvolvimento de demência em idosos que já apresentavam predisposição ao declínio cognitivo.

\section{CONCLUSÃO}

Existe uma clara associação entre o delirium e a demência/ declínio cognitivo, seja o delirium agindo como fator de risco para o desenvolvimento de um novo quadro de demência ou como fator agravante quando a demência já se encontra estabelecida em pacientes idosos. Novas pesquisas utilizando biomarcadores e tipos específicos de demência poderiam ser úteis para esclarecer os mecanismos neurobiológicos que envolvem essas duas entidades.

Considerando a prática clínica e o envelhecimento da população, torna-se imprescindível a investigação sistemática e o reconhecimento desses quadros, visto que diagnóstico e tratamento precoce poderiam evitar desfechos piores principalmente na qualidade de vida e funcionalidade do idoso.

\section{CONTRIBUIÇÕES INDIVIDUAIS}

Natália Mota S. Chagas - Escreveu a primeira versão da revisão e realizou a revisão dos artigos e a finalização do manuscrito.

Daniel G. Suzuki Borges - Contribuiu na escrita, revisão dos artigos e busca dos artigos.

Marcos Hortes N. Chagas - Supervisionou a busca de artigos e contribuiu na escrita e finalização do manuscrito.

\section{CONFLITOS DE INTERESSE}

Todos os autores declararam não apresentar conflitos de interesse.

\section{REFERÊNCIAS}

1. American Psychiatric Association. Diagnostic and statistical manual of mental disorders. $5^{\text {th }}$ ed. Washington DC: American Psychiatric Press; 2013

2. McCusker J, Cole M, Dendukuri N, Belzile E, Primeau F. Delirium in older medical inpatients and subsequent cognitive and functional status: a prospective study. CMAJ. 2001;165(5):575-83.

3. Krogseth M, Wyller TB, Engedal $K$, Julieb $\emptyset$ V. Delirium is an important predictor of incident dementia among elderly hip fracture patients. Dement Geriatr Cogn Disord. 2011;31(1):6370

4. van Munster BC, de Rooij SE. Delirium: a synthesis of current knowledge. Clin Med (Lond). 2014;14(2):192-5

5. Lorenzl S, Füsgen I, Noachtar S. Acute confusional States in the elderly -diagnosis and treatment. Dtsch Arztebl Int. 2012;109(21):391-9. 
6. Silverstein JH, Deiner SG. Perioperative delirium and its relationship to dementia. Prog Neuropsychopharmacol Biol Psychiatry. 2013;43:108-15.

7. Popp J. Delirium and cognitive decline: more than a coincidence. Curr Opin Neurol. 2013;26(6):634-9.

8. Fong TG, Davis D, Growdon ME, Albuquerque A, Inouye SK. The interface between delirium and dementia in elderly adults. Lancet Neurol. 2015;14(8):823-32.

9. Melkas S, Laurila JV, Vataja R, Oksala N, Jokinen H, Pohjasvaara T, et al. Post-stroke delirium in relation to dementia and long-term mortality. Int J Geriatr Psychiatry. 2012;27(4):401-8.

10. Bickel H, Gradinger R, Kochs E, Förstl H. High risk of cognitive and functional decline after postoperative delirium. A three-year prospective study. Dement Geriatr Cogn Disord. 2008;26(1):26-31.

11. van Rijsbergen MW, Oldenbeuving AW, Nieuwenhuis-Mark RE, Nys GM, Las SG, Roks G, et al. Delirium in acute stroke: a predictor of subsequent cognitive impairment? A two-year follow-up study. J Neurol Sci. 2011;306(1-2):138-42.
12. Davis DH, Muniz Terrera G, Keage H, Rahkonen T, Oinas M, Matthews FE, et al. Delirium is a strong risk factor for dementia in the oldest-old: a population-based cohort study. Brain. 2012;135(Pt 9):2809-16.

13. Lundström M, Edlund A, Bucht $G$, Karlsson S, Gustafson Y. Dementia after delirium in patients with femoral neck fractures. J Am Geriatr Soc. 2003;51(7):1002-6.

14. Kat MG, Vreeswijk R, de Jonghe JF, van der Ploeg T, van Gool WA, Eikelenboom P, et al. Long-term cognitive outcome of delirium in elderly hip surgery patients. A prospective matched controlled study over two and a half years. Dement Geriatr Cogn Disord. 2008;26(1):1-8.

15. Rahkonen T, Eloniemi-Sulkava U, Halonen P, Verkkoniemi A, Niinistö L, Notkola IL, et al. Delirium in the non-demented oldest old in the general population: risk factors and prognosis. Int J Geriatr Psychiatry. 2001;16(4):415-21.

16. Kalisvaart KJ, de Jonghe JF, Bogaards MJ, Vreeswijk R, Egberts TC, Burger BJ, et al. Haloperidol prophylaxis for elderly hip-surgery patients at risk for delirium: a randomized placebo-controlled study. J Am Geriatr Soc. 2005;53(10):1658-66. 